\title{
Fusion cross section measurements of astrophysical interest for light heavy ions systems within the STELLA project
}

\author{
Guillaume Fruet $^{1, \star}$, Sandrine Courtin ${ }^{1,2}$, David G. Jenkins ${ }^{3}$, Marcel Heine ${ }^{1}$, Daniele Montanari ${ }^{1,2}$, Luke G. Morris ${ }^{3}$, \\ Philip Adsley ${ }^{3,4}$, Christian Beck ${ }^{1}$, Serge Della Negra ${ }^{4}$, Florent Haas ${ }^{1}$, Fairouz Hammache ${ }^{4}$, Oliver S. Kirsebom ${ }^{5}$, \\ Anne Meyer ${ }^{4}$, Patrick H. Regan ${ }^{6,7}$, Matthias Rudiger ${ }^{6}$, Nicolas de Séréville ${ }^{4}$, and Christelle Stodel $^{8}$ \\ ${ }^{1}$ Université de Strasbourg, CNRS, IPHC UMR 7178, F-67000 Strasbourg, France \\ ${ }^{2}$ USIAS, Université de Strasbourg, F-67000 Strasbourg, France \\ ${ }^{3}$ Department of Physics, University of York, YO10 5DD York, United Kingdom \\ ${ }^{4}$ Institut de Physique Nucléaire d'Orsay, UMR8608, CNRS/IN2P3, Université Paris-Sud 11, F-91406 Orsay, France \\ ${ }^{5}$ Department of Physics and Astronomy, Aarhus University, DK-8000 Århus C, Denmark \\ ${ }^{6}$ Department of Physics, University of Surrey, GU2 7XH Guildford, United Kingdom \\ ${ }^{7}$ AIR Division, National Physical Laboratory, TW11 OLW Teddington, United Kingdom \\ ${ }^{8}$ Grand Accélérateur National d'lons Lourds, CNRS/IN2P3, F-14076 Caen, France
}

\begin{abstract}
This contribution is focused on the STELLA project (STELlar LAboratory), which aims at the measurement of fusion cross sections between light heavy ions like ${ }^{12} \mathrm{C}+{ }^{12} \mathrm{C},{ }^{12} \mathrm{C}+{ }^{16} \mathrm{O}$ or ${ }^{16} \mathrm{O}+{ }^{16} \mathrm{O}$ at deep subbarrier energies. The gamma-particle coincidence technique is used in order to reduce background contributions that become dominant for measurements in the nanobarn regime.

The experimental setup composed of an ultra high vacuum reaction chamber, a set of 3 silicon strip detectors, up to $36 \mathrm{LaBr}_{3}(\mathrm{Ce})$ scintillators from the UK FATIMA collaboration, and a fast rotating target system will be described. The ${ }^{12} \mathrm{C}+{ }^{12} \mathrm{C}$ fusion reaction has been studied from $\mathrm{E}_{\text {lab }}=11$ to $5.6 \mathrm{MeV}$ using STELLA at the Andromède facility in Orsay, France. Preliminary commissioning results are presented in this article.
\end{abstract}

\section{Introduction}

The measurement of fusion cross sections between light heavy ions like ${ }^{12} \mathrm{C},{ }^{16} \mathrm{O}$ or ${ }^{20} \mathrm{Ne}$ is at the center of interest to both nuclear physics and astrophysics. In particular, the ${ }^{12} \mathrm{C}+{ }^{12} \mathrm{C}$ fusion is a key reaction in the nucleosynthesis process during the $\mathrm{C}$ burning phase in the core of massive stars, in type Ia supernovae, and in superbursts from accreting neutron stars [1].

For reactions involving light ions, structural effects may seriously affect the fusion process. For instance, the ${ }^{12} \mathrm{C}-{ }^{12} \mathrm{C}$ system exhibits strong resonances from a few $\mathrm{MeV} / \mathrm{A}$ down to the sub-barrier regime, suggesting possible cluster formation in the compound nucleus [2], while an alternative explanation involves relatively large spacings and narrow widths of ${ }^{24} \mathrm{Mg}$ compound nucleus energy levels [3].

The fusion reaction mechanism has been widely studied in the past [4-6], and despite considerable efforts in both experimental and theoretical studies questions concerning the behaviour of such reactions at very low colliding energies remain open. Indeed, it can be seen in figure 1 that data show discrepancies among the various ex-

\footnotetext{
^e-mail: guillaume.fruet@iphc.cnrs.fr
}

periments with large uncertainties at the lowest measured energies. Moreover, extrapolations towards the astrophysical region differ by orders of magnitude [7].

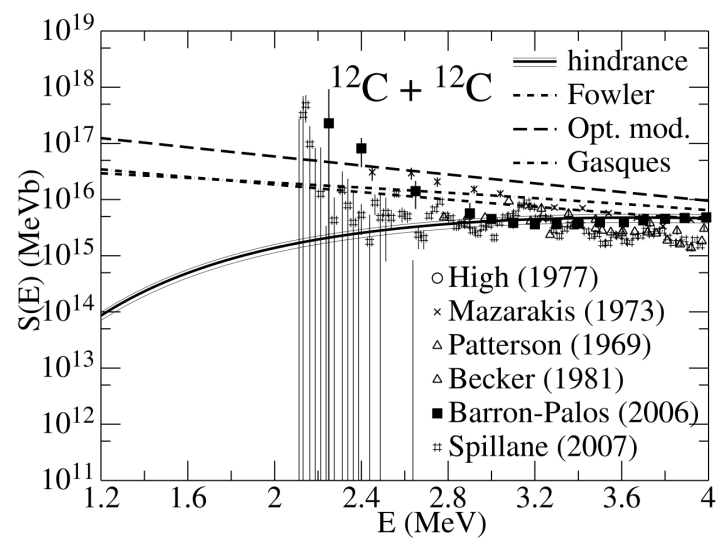

Figure 1. S-factor as a function of center of mass energy for the ${ }^{12} \mathrm{C}+{ }^{12} \mathrm{C}$ reaction. Theoretical calculations differ by orders of magnitude in the Gamow window $\mathrm{E}_{\mathrm{G}}=1.5 \pm 0.3 \mathrm{MeV}$ at $\mathrm{T}=5 \times$ $10^{8} \mathrm{~K}$ for this system.

The measurement technique will be presented in Sect. 2. The dedicated STELLA setup as well as technical developments will then be presented in Sect. 3. Fi- 
nally, preliminary results from the commissioning phase of STELLA will be discussed in Sect. 4.

\section{Experimental approach}

The ${ }^{12} \mathrm{C}+{ }^{12} \mathrm{C}$ fusion reaction has been studied at several beam energies around and below the Coulomb barrier $\left(\mathrm{E}_{\mathrm{C}} \sim 6.6 \mathrm{MeV}\right)$. In this regime, the three main exit channels are:

$$
\begin{aligned}
& { }^{12} \mathrm{C}+{ }^{12} \mathrm{C} \quad \rightarrow \quad{ }^{20} \mathrm{Ne}+\alpha \quad(Q=4.62 \quad \mathrm{MeV}), \\
& { }^{12} \mathrm{C}+{ }^{12} \mathrm{C} \rightarrow{ }^{23} \mathrm{Na}+\mathrm{p} \quad(Q=2.24 \quad \mathrm{MeV}), \\
& { }^{12} \mathrm{C}+{ }^{12} \mathrm{C} \rightarrow{ }^{23} \mathrm{Mg}+\mathrm{n} \quad(Q=-2.6 \quad \mathrm{MeV}) \text {. }
\end{aligned}
$$

At stellar temperature $\mathrm{T}=5 \times 10^{8} \mathrm{~K}$, the corresponding Gamow energy for ${ }^{12} \mathrm{C}+{ }^{12} \mathrm{C}$ is $\mathrm{E}_{\mathrm{G}}=1.5 \pm 0.3 \mathrm{MeV}$, below the neutron emission threshold. Thus, the exit channel (3) has not been studied within this work.

The detection of either light charged particles or $\gamma$ 's from the deexcitation of both residual nuclei ${ }^{20} \mathrm{Ne}$ and ${ }^{23} \mathrm{Na}$ has been widely used in the past to identify fusion events (see $[4,6,8]$ ).

Recently, it has been shown in Ref. [9] that the detection of the light charged particles in coincidence with the $\gamma$-ray from the heavy partner allows a very reliable selection of fusion events, due to a drastical rejection of background coming essentially from target contaminants $\left({ }^{13} \mathrm{C},{ }^{1} \mathrm{H},{ }^{2} \mathrm{H}, \ldots\right)$ or room background radiation.

For the present measurement, ${ }^{12} \mathrm{C}$ targets of 20,30 and $50 \mu \mathrm{g} / \mathrm{cm}^{2}$ are used. It is of great importance to avoid carbon build-up on them, a process that will affect precise determination of the reaction energy and probability by increasing the target thickness [10].

The differential cross section is then given by:

$$
\left(\frac{d \sigma}{d \Omega}\right)_{\mathrm{lab}}=\frac{N}{I \cdot \epsilon_{\gamma} \cdot \Delta \Omega \cdot \Delta t \cdot N_{t}},
$$

where $N$ is the number of coincidence events, $I$ the beam intensity, $\Delta t$ the acquisition time, $N_{t}$ the number of atoms in the target, $\Delta \Omega$ the solid angle covered by the particle detector, and $\epsilon_{\gamma}$ the $\gamma$ efficiency.

To clarify the evolution of the S-factor towards the Gamow window, fusion cross sections below the nanobarn range should be measured. One possibility to obtain sufficient statistics is to increase the beam intensity, typically at values $\geq 1 \mathrm{p} \mu \mathrm{A}$. Under such conditions, the usual thin fixed targets have a relatively short lifetime of few hours and an improved target system would be useful.

\section{Technical developments}

\subsection{Reaction chamber and particle detectors}

A reaction chamber has been designed and machined at IPHC-CNRS Strasbourg, France. The bottom part consists of a stainless steel cylinder used to accomodate the particle detector support, feedthrough and the pumping system. The upper part is a $2.5 \mathrm{~mm}$ thick aluminium dome of $20 \mathrm{~cm}$ diameter around which are placed $\mathrm{LaBr}_{3}(\mathrm{Ce})$ scintillators for gamma detection.

Light charged particles like alphas and protons are detected using annular double-sided silicon strip detectors (DSSD), S1 and S3 design by Micron Semiconductor. These detectors are divided into 16 and 24 rings, respectively.

A PCB to support the silicon chips has been developed and built using R00403C material, which is known for low outgazing to prevent carbon build-up in targets.

Along with this development, a new pin connection system has been conceived. It allows to read signals of detectors from below the target plane, thus reducing material budget inside the reaction chamber. In the arrangment configuration seen in figure 2 , the total solid angle coverage is $\sim 24 \%$ of $4 \pi$.

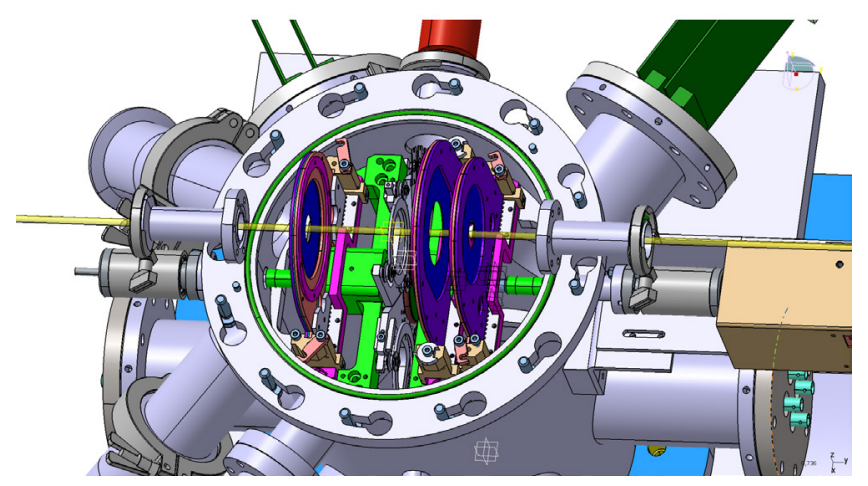

Figure 2. Mechanical drawing of the reaction chamber and the arrangment of annular Si-strip detectors around the target which is at the center of the chamber. The beam is going from right to left.

The resolution of these detectors is about $35-40 \mathrm{keV}$ (FWHM) for particle energies around $5.5 \mathrm{MeV}$ giving sufficient resolution to separate the different energies associated to protons and alphas coming from the fusion reaction. Aluminium foils of $0.8 \mu \mathrm{m}$ thickness protect detectors at backward angles from low energy electrons emitted from the target, while a $10 \mu \mathrm{m}$ foil prevents intense elastically scattered ${ }^{12} \mathrm{C}$ beam to reach the forward detector.

To obtain an absolute normalisation for the fusion cross section two $\mathrm{Si}$ surface barrier detectors are used to measure scattered beam. They are placed symetrically at an angle $\theta_{\text {lab }}=45^{\circ}$ with respect to the beam axis, where the Mott cross section has a rather flat and energy-independent local maximum decreasing systematic uncertainties associated with the precise location of the monitors. A Faraday cup after the chamber was also used to control the beam intensity.

The signals from the Si detectors are processed using a data acquisition system based on the $\mu$ TCA technology. It is a digital time stamp acquisition system supporting 96 
channels, and a dedicated card distributes an external 125 $\mathrm{MHz}$ frequency signal used to synchronise the clocks of particle and $\gamma$ cards to reconstruct coincident events offline.

\subsection{Gamma detectors}

At bombarding energies below the Coulomb barrier, the states that can be populated in ${ }^{20} \mathrm{Ne}$ and ${ }^{23} \mathrm{Na}$ exhibit a deexcitation pattern which cascades essentially through their first excited state $[11,12]$. The energies of the corresponding $\gamma$ transitions are $\mathrm{E}_{\gamma}=1634 \mathrm{keV}$ and $\mathrm{E}_{\gamma}=$ $440 \mathrm{keV}$ for exit channel (1) and (2), respectively.

Novel generation scintillators are widely used in nuclear physics as they provide sufficient energy resolution combined with a high detection efficiency. In this project, $\mathrm{LaBr}_{3}(\mathrm{Ce})$ detectors from the UK FATIMA collaboration have been combined with our system. Details concerning these detectors can be found in Ref. [13].

Comprehensive Geant 4 simulations have been carried out to retrieve the highest photopeak efficiency for the detection of $\gamma$ lines of interest. A cylindrical setup where all the detectors are facing the beam line has been constructed. A mechanical drawing of the supporting structure is shown figure 3 and a detailed description of the efficiency studies can be found in Ref. [14].

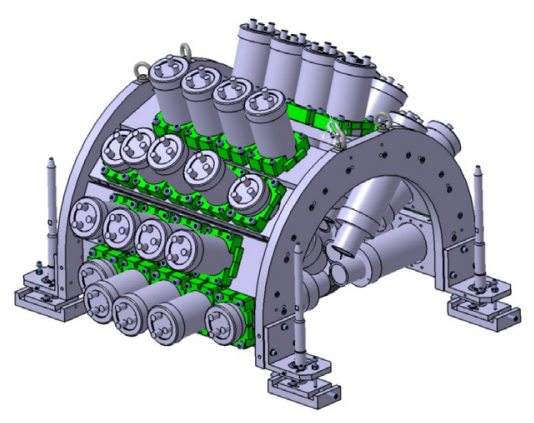

Figure 3. Cylindrical supporting structure of the $36 \mathrm{LaBr}_{3}(\mathrm{Ce})$ crystals. The detectors face the beam line and are arranged in the most compact configuration.

In the optimal configuration where $36 \mathrm{LaBr}_{3}(\mathrm{Ce})$ are used the photopeak efficiency is $\epsilon \sim 3 \%$ for $\mathrm{E}_{\gamma}=1634$ $\mathrm{keV}$, and $\epsilon \sim 8 \%$ for $\mathrm{E}_{\gamma}=440 \mathrm{keV}$.

The detectors self activity, which originates from the decay of the ${ }^{138} \mathrm{La}$ isotope as well as the ${ }^{224} \mathrm{Ac}$ contaminant, produces a constant background in $\gamma$ spectra that is irrelevant in coincidence analysis. This can be used to perform an online calibration of the spectra allowing to correct for the gain drift of the PMTs that may occur with change of room temperature. A preliminary framework for this procedure is also presented in Ref. [14].

\subsection{Target developments}

In order to avoid carbon build-up on targets, several efforts have been made to guarantee an ultra high vacuum in the reaction chamber. Thus, only compatible materials have been used inside STELLA, like suited high vacuum connectors and PCB for DSSDs.

The pumping system is composed of a primary dry pump and a cryopump cooled down to a temperature of about $15 \mathrm{~K}$. The latter has a sufficient diameter to cover the entire volume of the cylindrical part of the chamber, and a pressure of $\sim 3 \times 10^{-8}$ mbar is obtained close to the target position.

A serious limitation while using thin carbon targets is their lifetime when beam intensity is higher than $1 \mathrm{p} \mu \mathrm{A}$. One possibility to overcome this difficulty is to use a fast rotating target system where the beam spot location is distributed along a path on the target surface. This enables better heat dissipation which is one of the cause of the target breaking process.

In collaboration with GANIL, a dedicated rotating target system has then been developed for the STELLA project and tested under beam at the end of the commissioning phase. Details of the apparatus will be given in a forthcoming technical paper.

\subsection{The Andromède facility}

For the first campaign, the STELLA station has been installed to the Andromède facility [15] in Orsay, France. Andromède is a $4 \mathrm{MV}$ Pelletron accelerator which can provide various beams from light ions such as ${ }^{4} \mathrm{He},{ }^{12} \mathrm{C}$, ${ }^{16} \mathrm{O}$, up to heavy Au-cluster or $\mathrm{CH}_{4}$ molecules.

STELLA was placed at the dedicated $90^{\circ}$ line where the presence of a magnetic dipole ensures the delivery of a high purity ${ }^{12} \mathrm{C}$ beam. The accelerator ran in very stable energy and intensity conditions during the whole data taking.

During the experiment, we used ${ }^{12} \mathrm{C}^{3+}$ and ${ }^{12} \mathrm{C}^{2+}$ beams from $\mathrm{E}_{\mathrm{lab}}=11$ to $5.6 \mathrm{MeV}$ impinging onto fix targets of 20,30 and $50 \mu \mathrm{g} / \mathrm{cm}^{2}$ with beam intensity varying from $40 \mathrm{pnA}$ to $450 \mathrm{pnA}$. Preliminary results are presented in the next section.

\section{Preliminary results}

A typical matrix of the particle energy as a function of ring number of a DSSD located at backward angles $\left(148^{\circ} \leq \theta_{\text {lab }} \leq 168^{\circ}\right)$ is shown in figure 4 . This spectrum was obtained at a beam energy of $11 \mathrm{MeV}$ and the solid lines correspond to kinematics calculations for the different protons and alphas $\left(\mathrm{p}_{i}\right.$ and $\left.\alpha_{i}\right)$ associated to excited states in the heavy reaction ejectile. No contaminant contribution is present in the spectrum at this energy, where the total fusion cross section is $\sim 20 \mathrm{mb}$.

The corresponding single $\gamma$ spectrum is shown in figure 5. This is the sum over the 28 detectors used during 


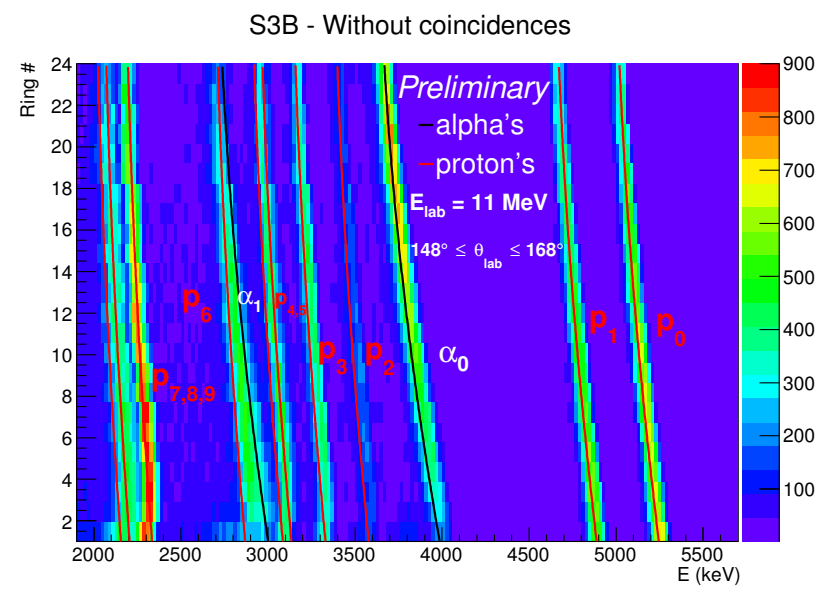

Figure 4. Angular distribution of particles obtained with a DSSD located at backward angles. The solid lines are kinematics calculations at $\mathrm{E}_{\text {lab }}=11 \mathrm{MeV}$ for the proton (red) and alpha (black) exit channels.

the first phase of the experiment.

As mentioned in Sect. 3.2, a continuous background is visible in the single $\gamma$ spectrum mainly due to $\mathrm{LaBr}_{3}$ self activity and gamma transitions originating from the first excited states of ${ }^{20} \mathrm{Ne}$ and ${ }^{23} \mathrm{Na}$ are masked by this contribution.

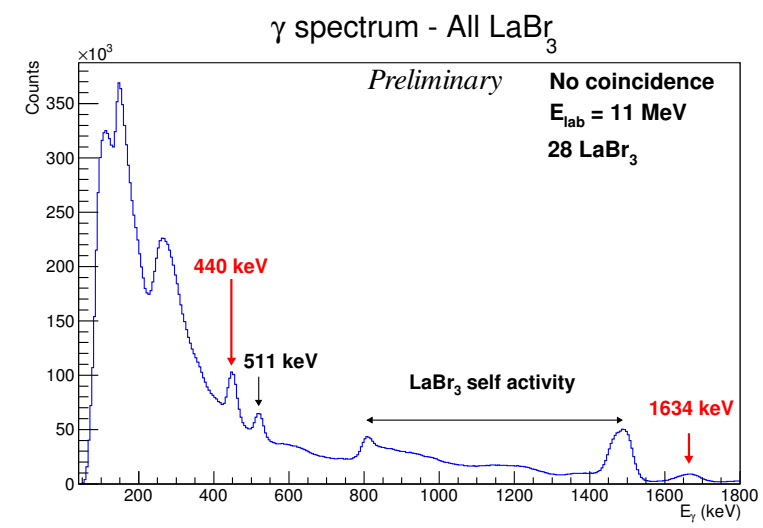

Figure 5. Gamma spectrum summed over $28 \mathrm{LaBr}_{3}$ obtained at $\mathrm{E}_{\text {lab }}=11 \mathrm{MeV}$. The self activity as well as the two important $\gamma$ lines at 440 and $1634 \mathrm{keV}$ from the $\mathrm{C}+\mathrm{C}$ fusion are indicated by arrows.

Nevertheless, this background in the single $\gamma$ spectrum can be suppressed when analysing coincident events. A typical spectrum of the detection time difference between $\gamma$ 's and particles is shown in figure 6 where a well defined peak around $350 \mathrm{~ns}$ with few random coincidences spread all over the spectrum is visible. This allows a reliable selection of coincident events.

As an example, the effect of requiring a $\gamma$ coincidence in the particle spectrum from a DSSD located at backward angles when gating around $\mathrm{E}_{\gamma}=440 \mathrm{keV}$ is depicted in figure 7 , where single and coincident spectra are shown

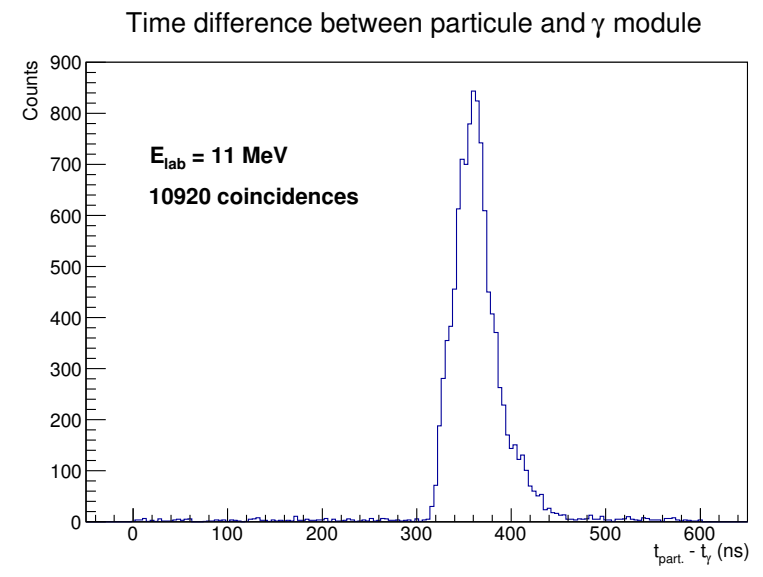

Figure 6. Time difference between $\gamma$ and particle detection. A background free coincident peak is clearly visible.

together. The $\mathrm{p}_{0}$ and $\alpha_{0}$ exit channels are associated to

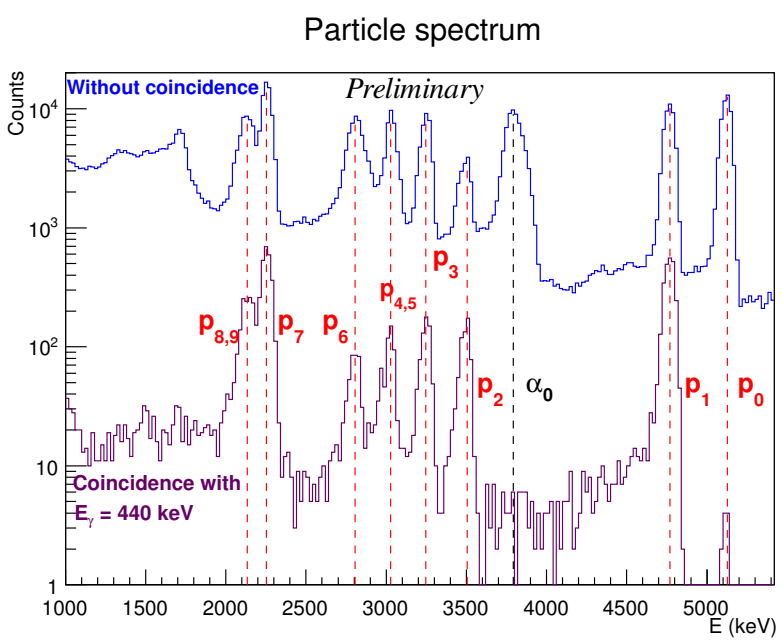

Figure 7. Single particle spectrum (upper part) and the corresponding one obtained by gating around $\mathrm{E}_{\gamma}=440 \mathrm{keV}$ (lower part) for a DSSD located at backward angles. The $\mathrm{p}_{0}$ and $\alpha_{0}$ exit channels, which have no true coincidence with $\gamma$ 's, are effectively suppressed in the coincident spectrum.

the population of the ground state of the heavy partner ${ }^{23} \mathrm{Na}$ and ${ }^{20} \mathrm{Ne}$, respectively. No coincident gammas are expected and their contributions are effectively suppressed in the coincident spectrum.

In figure 7 , the protons associated to the different excited states in ${ }^{23} \mathrm{Na}$ are separated enough to obtain partial fusion cross sections after correcting for the decay branching ratios tabulated in Ref. [12].

From the spectra displayed in figure 7, a first experimental value can be obtained for the detection efficiency of $\mathrm{E}_{\gamma}=440 \mathrm{keV}$ by taking the ratio of integrated coincident and single $\mathrm{p}_{1}$ peaks. This gives a result of about $6 \%$, which is in agreement with Geant 4 simulation of the 28 detectors setup used during the experiment. Further checks 
will be done by comparing simulation results to $\gamma$ source runs where a ${ }^{152} \mathrm{Eu}$ source has been utilized.

\section{Summary}

A new dedicated experimental station (STELLA) has been developed and built in IPHC-CNRS, Strasbourg, to measure fusion cross sections of light heavy ions. It makes use of the $\gamma$-particle coincidence technique to ensure reliable selection of fusion events at deep sub-barrier energies.

During the first campaign, STELLA has been coupled to the $\mathrm{LaBr}_{3}$ detectors from the UK FATIMA collaboration and installed at the Andromède facility in Orsay, France, to study the ${ }^{12} \mathrm{C}+{ }^{12} \mathrm{C}$ fusion reaction from $\mathrm{E}_{\mathrm{lab}}=11$ to 5.6 $\mathrm{MeV}$.

Identification of the various exit channels of the reaction has been proven possible using both single and coincident particle spectra. This allows to take into account in the total fusion cross section the feeding of the ground and excited states in the ${ }^{23} \mathrm{Na}$ and ${ }^{20} \mathrm{Ne}$ exit channels.

In collaboration with GANIL, a fast rotating target system has been developed and tested under beam. With this apparatus, a beam intensity of $\sim 5 \mathrm{p} \mu \mathrm{A}$ may be used in the future. Then, considering the measured resonance of Spillane et al. at $\mathrm{E}_{\mathrm{cm}}=2.14 \mathrm{MeV}$ [16] a statistical uncertainty of $30 \%$ can be obtained in about 3 days, whereas several weeks are needed following Gasques et al. extrapolation [17].

\section{Acknowledgement}

We wish to thank G. Frémont (GANIL) and M. Loriggiola (LNL, Legnaro) for preparation of ${ }^{12} \mathrm{C}$ targets for this commissioning run.

\section{References}

[1] L.R. Gasques et al., PRC 76, 035802 (2007)

[2] R.G. Stokstad et al., PRL 37, 888891 (1976)

[3] C.L. Jiang et al., PRL 110, 072701 (2013)

[4] L.J. Patterson et al., The Astr. J. 157, 367373 (1969)

[5] B. Cujec et al., NPA 266, 461493 (1976)

[6] H.W. Becker et al., ZPA 303, 305312 (1981)

[7] C.L. Jiang et al., PRC 75, 015803 (2007)

[8] K.U. Kettner et al., PRL 38, 337340 (1977)

[9] C.L. Jiang et al., NIMA 682, 1215 (2012)

[10] E.F. Aguilera et al., PRC 73, 064601 (2006)

[11] D.R. Tilley et al., NPA 636, 249 (1998)

[12] R.B. Firestone, Nuclear Data Sheets 108, 1 (2007)

[13] O.J. Roberts et al., NIMA 748, 9195 (2014)

[14] M. Heine et al., JoP: Conf. Ser. 763, 012005 (2016)

[15] M.J. Eller et al., NIMB 365, 367370 (2015)

[16] T. Spillane et al., PRL 98, 122501 (2007)

[17] L.R. Gasques et al., PRC 72, 025806 (2005) 